\title{
A system of nonlinear set valued variational inclusions
}

\author{
Yong-Kun Tang ${ }^{1}$, Shih-sen Chang ${ }^{1 *}$ and Salahuddin Salahuddin ${ }^{2}$
}

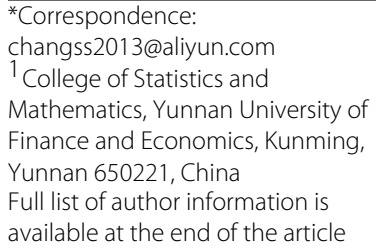

\begin{abstract}
In this paper, we studied the existence theorems and techniques for finding the solutions of a system of nonlinear set valued variational inclusions in Hilbert spaces. To overcome the difficulties, due to the presence of a proper convex lower semicontinuous function $\phi$ and a mapping $g$ which appeared in the considered problems, we have used the resolvent operator technique to suggest an iterative algorithm to compute approximate solutions of the system of nonlinear set valued variational inclusions. The convergence of the iterative sequences generated by algorithm is also proved.
\end{abstract}

AMS Mathematics subject classification: $49 \mathrm{~J} 40 ; 47 \mathrm{HO6}$

Keywords: System of nonlinear set valued variational inclusions; Lipschitz continuity; Resolvent operators; Iterative sequences; Hilbert spaces

\section{Introduction}

It is well known that variational inequality theory and complementarity problems are very powerful tools of current mathematical technology. In recent years, the classical variational inequality and complementarity problems have been extended and generalized to study a large variety of problems arising in economics, control problems, contact problems, mechanics, transportation, equilibrium problems, optimization theory, nonlinear programming, transportation equilibrium and engineering sciences, see (Aubin 1982; Baiocchi and Capelo 1984; Chang 1984; Giannessi and Maugeri 1995). Hassouni and Moudafi 2001 introduced and studied a class of mixed type variational inequalities with single valued mappings which was called variational inclusions. Since many authors have obtained important extension generalizations of the results in (Hassouni and Moudafi 2001) from various directions, see (Agarwal et al. 2011; Fang et al. 2005; Kassay and Kolumban 2000; Petrot 2010). Verma 1999; 2001a introduced and studied some system of variational inequalities with iterative algorithms to compute approximate solutions in Hilbert spaces.

Inspired and motivated by the research work going on this field, in this works, the methods for finding the common solutions of a system of nonlinear set valued variational inclusions involving different nonlinear operators and fixed point problem are considered and studied, via proximal method in the framework of Hilbert spaces.

Since the problems of a system of a nonlinear set valued variational inequalities and fixed point are both important, the results present in this paper are useful and can be viewed as an improvement and extension of the previously known results appearing in

(c) 2014 Tang et al.; licensee Springer. This is an Open Access article distributed under the terms of the Creative Commons

Attribution License (http://creativecommons.org/licenses/by/4.0), which permits unrestricted use, distribution, and reproduction in any medium, provided the original work is properly credited. 
literature, which are improves the results of Chang et al. 2007 and also extends the results of Verma 2001b; 2002, Ahmad and Salahuddin 2012, Ding and Luo 2000, Inchan and Petrot 2011, Kim and Kim 2004, Kim and Hu 2008, Nie et al. 2003 and Suantai and Petrot 2011, etc.

Let $H$ be a real Hilbert space whose inner product and norm are denoted by $\langle\cdot, \cdot\rangle$ and $\|\cdot\|$ respectively and $K$ be a nonempty closed convex subset of $H$. Let $C B(H)$ be the family of all nonempty closed convex and bounded sets in $H$ and $\phi: H \rightarrow(-\infty,+\infty)$ be a proper convex lower semicontinuous function on $H$. Let $N_{i}: H \times H \rightarrow H$ be a nonlinear function, $g_{i}: K \rightarrow H$ be a nonlinear operator, $A_{i}, B_{i}: K \rightarrow C B(H)$ be the nonlinear set valued mappings and let $r_{i}$ be a fixed positive real number for each $i=1,2,3$. Set $\Xi=\left\{N_{1}, N_{2}, N_{3}\right\}, \mathfrak{A}=\left\{A_{1}, A_{2}, A_{3}\right\}, \mathfrak{B}=\left\{B_{1}, B_{2}, B_{3}\right\}, \wedge=\left\{g_{1}, g_{2}, g_{3}\right\}$. The system of nonlinear set valued variational inclusions involving three different nonlinear operators is defined as follows:

Find $\left(x^{*}, y^{*}, z^{*}\right) \in H \times H \times H, u_{3}^{*} \in A_{3}\left(x^{*}\right), v_{3}^{*} \in B_{3}\left(x^{*}\right), u_{2}^{*} \in A_{2}\left(z^{*}\right), v_{2}^{*} \in B_{2}\left(z^{*}\right), u_{1}^{*} \in$ $A_{1}\left(y^{*}\right), v_{1}^{*} \in B_{1}\left(y^{*}\right)$, such that

$$
\left\{\begin{array}{l}
\left\langle r_{1} N_{1}\left(u_{1}^{*}, v_{1}^{*}\right)+g_{1}\left(x^{*}\right)-g_{1}\left(y^{*}\right), g_{1}(x)-g_{1}\left(x^{*}\right)\right\rangle-r_{1} \phi\left(g_{1}\left(x^{*}\right)\right)+r_{1} \phi\left(g_{1}(x)\right) \geq 0, \quad g_{1}(x) \in K, \\
\left\langle r_{2} N_{2}\left(u_{2}^{*}, v_{2}^{*}\right)+g_{2}\left(y^{*}\right)-g_{2}\left(z^{*}\right), g_{2}(x)-g_{2}\left(y^{*}\right)\right\rangle-r_{2} \phi\left(g_{2}\left(y^{*}\right)\right)+r_{2} \phi\left(g_{2}(x)\right) \geq 0, \quad g_{2}(x) \in K, \\
\left\langle r_{3} N_{3}\left(u_{3}^{*}, v_{3}^{*}\right)+g_{3}\left(z^{*}\right)-g_{3}\left(x^{*}\right), g_{3}(x)-g_{3}\left(z^{*}\right)\right\rangle-r_{3} \phi\left(g_{3}\left(z^{*}\right)\right)+r_{3} \phi\left(g_{3}(x)\right) \geq 0, \quad g_{3}(x) \in K .
\end{array}\right.
$$

We denote the set of all solutions $\left(x^{*}, y^{*}, z^{*}, u_{1}^{*}, v_{1}^{*}, u_{2}^{*}, v_{2}^{*}, u_{3}^{*}, v_{3}^{*}\right)$ of problem (1) by $\operatorname{SNSVVID}(\Xi, \mathfrak{A}, \mathfrak{B}, \wedge, K)$.

We first recall some basic concepts and well known results.

Definition 1. A mapping $g: H \rightarrow H$ is said to be

(i) monotone, if

$$
\langle g(x)-g(y), x-y\rangle \geq 0 \quad \forall x, y \in H ;
$$

(ii) strictly monotone, if $g$ is monotone and

$$
\langle g(x)-g(y), x-y\rangle=0 \text { if and only if } x=y ;
$$

(iii) $v$-strongly monotone, if there exists a constant $v>0$ such that

$$
\langle g(x)-g(y), x-y\rangle \geq v\|x-y\|^{2}, \quad \forall x, y \in H ;
$$

(iv) Lipschitz continuous, if there exists a constant $v>0$ such that

$$
\|g(x)-g(y)\| \leq v\|x-y\|, \forall x, y \in H .
$$

Definition 2. A set valued mapping $A: H \rightarrow 2^{H}$ is said to be v-strongly monotone, if there exists a constant $v>0$ such that

$$
\left\langle w_{1}-w_{2}, x-y\right\rangle \geq v\|x-y\|^{2}, \quad \forall x, y \in H, w_{1} \in A(x), w_{2} \in A y .
$$

Definition 3. A set valued mapping $A: H \rightarrow C B(H)$ is said to be $\tau$-Lipschitz continuous if there exists a constant $\tau>0$ such that

$$
\mathcal{H}(A x, A y) \leq \tau\|x-y\|, \quad \forall x, y \in H,
$$

where $\mathcal{H}(\cdot, \cdot)$ is the Hausdorff metric on $C B(\mathcal{H})$. 
Definition 4. (Brezis 1973)

If $M$ is maximal monotone operator on $H$ then for any $\lambda>0$ the resolvent operator associated with $M$ is defined by

$$
J_{M}(x)=(I+\lambda M)^{-1}(x), \forall x \in H .
$$

It is well know that a monotone operator is maximal iff its resolvent operator is defined every where. Furthermore the resolvent operator is single valued and nonexpansive. In particular the subdifferential $\partial \phi$ of a proper convex lower semicontinuous function $\phi: H \rightarrow(-\infty,+\infty)$ is a maximal monotone operator.

Lemma 1. (Brezis 1973) The points $u, z \in H$ satisfies the inequality

$$
\langle u-z, x-u\rangle+\lambda \phi(x)-\lambda \phi(u) \geq 0, \quad \forall x \in H,
$$

if and only if

$$
u=J_{\phi}^{\lambda}(z),
$$

where $J_{\phi}^{\lambda}=(I+\lambda \partial \phi)^{-1}$ is a resolvent operator and $\lambda>0$ is a constant.

For any $x, y \in H, J_{\phi}^{\lambda}$ is nonexpansive, i.e.,

$$
\left\|J_{\phi}^{\lambda}(x)-J_{\phi}^{\lambda}(y)\right\| \leq\|x-y\|, \forall x, y \in H .
$$

Assume that $g: H \rightarrow H$ is a surjective mapping and from Lemma 1 and (1) we have the following proximal point problem:

$$
\left\{\begin{array}{l}
g_{1}\left(x^{*}\right)=J_{\phi}^{r_{1}}\left[g_{1}\left(y^{*}\right)-r_{1} N_{1}\left(u_{1}^{*}, v_{1}^{*}\right)\right], \\
g_{2}\left(y^{*}\right)=J_{\phi}^{r_{2}}\left[g_{2}\left(z^{*}\right)-r_{2} N_{2}\left(u_{2}^{*}, v_{2}^{*}\right)\right], \\
g_{3}\left(z^{*}\right)=J_{\phi}^{r_{3}}\left[g_{3}\left(x^{*}\right)-r_{3} N_{3}\left(u_{3}^{*}, v_{3}^{*}\right)\right],
\end{array}\right.
$$

provided $K \subset g_{i}(H)$ for each $i=1,2,3$.

Lemma 2. (Weng 1991)

Let $\left\{a_{n}\right\},\left\{b_{n}\right\}$ and $\left\{c_{n}\right\}$ be three sequences of nonnegative real numbers such that

$$
a_{n+1} \leq\left(1-t_{n}\right) a_{n}+b_{n}+c_{n} \forall n>n_{0},
$$

where $n_{0}$ is a nonnegative integer, $\left\{t_{n}\right\}$ is a sequence in $(0,1)$ with $\sum_{n=0}^{\infty} t_{n}=+\infty$, $\lim _{n \rightarrow \infty} b_{n}=0\left(t_{n}\right)$ and $\sum_{n=0}^{\infty} c_{n}<+\infty$. Than $a_{n} \rightarrow 0$ as $n \rightarrow+\infty$.

Definition 5. Let $A, B: H \rightarrow 2^{H}$ be set valued mappings and $N: H \times H \rightarrow H$ be a nonlinear mapping.

(i) $\quad N$ is said to be $A$-strongly monotone with respect to the first argument, if there exists a constant $v>0$ such that for all $x, y \in H$

$$
\left\langle N\left(u_{1}, w\right)-N\left(u_{2}, w\right), x-y\right\rangle \geq v\|x-y\|^{2} \forall u_{1} \in A(x), u_{2} \in A(y), w \in H ;
$$

(ii) $\quad N$ is said to be $B$-relaxed monotone with respect to the second argument, if there exists a constant $\xi>0$ such that for all $x, y \in H, v_{1} \in B(x), v_{2} \in B(y)$

$$
\left\langle N\left(u, v_{1}\right)-N\left(u, v_{2}\right), x-y\right\rangle \geq-\xi\|x-y\|^{2}, \forall u \in H .
$$

\section{Main results}

We begin with some observations which are related to the problem (1). 
Remark 1. If $\left(x^{*}, y^{*}, z^{*}\right) \in \operatorname{SNSVVID}(\Xi, \mathfrak{A}, \mathfrak{B}, \wedge, K)$, by (2) we have that

$$
x^{*}=x^{*}-g_{1}\left(x^{*}\right)+J_{\phi}^{r_{1}}\left[g_{1}\left(y^{*}\right)-r_{1} N_{1}\left(u_{1}^{*}, v_{1}^{*}\right)\right] .
$$

provided $K \subset g_{1}(H)$

Consequently if $S$ is a Lipschitz mapping such that $x^{*} \in F(S)$, then it follows from (3) that

$$
x^{*}=S\left(x^{*}\right)=S\left(x^{*}-g_{1}\left(x^{*}\right)+J_{\phi}^{r_{1}}\left[g_{1}\left(y^{*}\right)-r_{1} N_{1}\left(u_{1}^{*}, v_{1}^{*}\right)\right]\right) .
$$

By virtue of (4) and Nadler's Theorem (Nadler 1969), we suggest the following iterative algorithm.

Algorithm 1 Let $\epsilon_{n}$ be a sequence of nonnegative real number with $\epsilon_{n} \rightarrow 0$ as $n \rightarrow \infty$. Let $r_{1}, r_{2}, r_{3}$ be three given positive real numbers in $(0,1)$. For arbitrary chosen initial $x_{0} \in H$, compute the sequences $\left\{x_{n}\right\},\left\{y_{n}\right\}$ and $\left\{z_{n}\right\}$ in $H$, such that

$$
\left\{\begin{array}{l}
g_{3}\left(z_{n}\right)=J_{\phi}^{r_{3}}\left[g_{3}\left(x_{n}\right)-r_{3} N_{3}\left(u_{n, 3}, v_{n, 3}\right)\right], \\
g_{2}\left(y_{n}\right)=J_{\phi}^{r_{2}}\left[g_{2}\left(z_{n}\right)-r_{2} N_{2}\left(u_{n, 2}, v_{n, 2}\right)\right], \quad \forall n \geq 1 \\
x_{n+1}=\left(1-\alpha_{n}\right) x_{n}+\alpha_{n} S\left(x_{n}-g_{1}\left(x_{n}\right)+J_{\phi}^{r_{1}}\left[g_{1}\left(y_{n}\right)-r_{1} N_{1}\left(u_{n, 1}, v_{n, 1}\right)\right]\right),
\end{array}\right.
$$

where

$$
\left\{\begin{array}{l}
u_{n, 3} \in A_{3}\left(x_{n}\right), u_{n-1,3} \in A_{3}\left(x_{n-1}\right):\left\|u_{n, 3}-u_{n-1,3}\right\| \leq\left(1+\epsilon_{n}\right) \mathcal{H}\left(A_{3}\left(x_{n}\right), A_{3}\left(x_{n-1}\right)\right) \\
v_{n, 3} \in B_{3}\left(x_{n}\right), v_{n-1,3} \in B_{3}\left(x_{n-1}\right):\left\|v_{n, 3}-v_{n-1,3}\right\| \leq\left(1+\epsilon_{n}\right) \mathcal{H}\left(B_{3}\left(x_{n}\right), B_{3}\left(x_{n-1}\right)\right) \\
u_{n, 2} \in A_{2}\left(z_{n}\right), u_{n-1,2} \in A_{2}\left(z_{n-1}\right):\left\|u_{n, 2}-u_{n-1,2}\right\| \leq\left(1+\epsilon_{n}\right) \mathcal{H}\left(A_{2}\left(z_{n}\right), A_{2}\left(z_{n-1}\right)\right) \\
v_{n, 2} \in B_{2}\left(z_{n}\right), v_{n-1,2} \in B_{2}\left(z_{n-1}\right):\left\|v_{n, 2}-v_{n-1,2}\right\| \leq\left(1+\epsilon_{n}\right) \mathcal{H}\left(B_{2}\left(z_{n}\right), B_{2}\left(z_{n-1}\right)\right) \\
u_{n, 1} \in A_{1}\left(y_{n}\right), u_{n-1,1} \in A_{1}\left(y_{n-1}\right):\left\|u_{n, 1}-u_{n-1,1}\right\| \leq\left(1+\epsilon_{n}\right) \mathcal{H}\left(A_{1}\left(y_{n}\right), A_{1}\left(y_{n-1}\right)\right) \\
v_{n, 1} \in B_{1}\left(y_{n}\right), v_{n-1,1} \in B_{1}\left(y_{n-1}\right):\left\|v_{n, 1}-v_{n-1,1}\right\| \leq\left(1+\epsilon_{n}\right) \mathcal{H}\left(B_{1}\left(y_{n}\right), B_{1}\left(y_{n-1}\right)\right)
\end{array}\right.
$$

and $\left\{\alpha_{n}\right\}$ is a sequence in $(0,1)$ and $S: H \rightarrow H$ is a mapping.

Theorem 1. Let $K$ be a nonempty closed and convex subset of a real Hilbert space $H$ and $\phi: H \rightarrow(-\infty,+\infty)$ be a proper convex lower semicontinuous function. Let $A_{i}: H \rightarrow 2^{H}$ be a $\mu_{i}$-Lipschitz continuous mapping with $\mu_{i}<1$ and $B_{i}: H \rightarrow 2^{H}$ be a $\sigma_{i}$-Lipschitz continuous mapping with $\sigma_{i}<1, i=1,2$, 3. Let $N_{i}: H \times H \rightarrow H$ be a $\rho_{i}$-Lipschitz continuous with respect to the first variable and $\eta_{i}$-Lipschitz continuous with respect to the second variable and $N_{i}$ be $A_{i}$-strongly monotone with constant $v_{i}>0$ and $B_{i}$-relaxed monotone with constant $\xi_{i}>0, i=1,2,3$. Let $g_{i}: H \rightarrow H$ be a $\lambda_{i}$-strongly monotone and $\gamma_{i}$-Lipschitz continuous mapping, $i=1,2$, 3. Let $S: H \rightarrow H$ be a $\tau$-Lipschitz continuous mapping with $0<\tau \leq 1$. If SNSVVID $(\Xi, \mathfrak{A}, \mathfrak{B}, \wedge, K) \cap F(S) \neq \emptyset$, and the following conditions are satisfied:

(i)

$$
h_{i} \in\left[0, \frac{\left(\rho_{i} \mu_{i}+\eta_{i} \sigma_{i}\right)-\sqrt{\left(\rho_{i} \mu_{i}+\eta_{i} \sigma_{i}\right)^{2}-\left(v_{i}-\xi_{i}\right)^{2}}}{2\left(\rho_{i} \mu_{i}+\eta_{i} \sigma_{i}\right)}\right) \bigcup\left[\frac{\left(\rho_{i} \mu_{i}+\eta_{i} \sigma_{i}\right)+\sqrt{\left(\rho_{i} \mu_{i}+\eta_{i} \sigma_{i}\right)^{2}-\left(v_{i}-\xi_{i}\right)^{2}}}{2\left(\rho_{i} \mu_{i}+\eta_{i} \sigma_{i}\right)}, 1\right)
$$

where $h_{i}=\sqrt{1-2 \lambda_{i}+\gamma_{i}^{2}}, i=1,2,3$; 
(ii)

$$
\left|r_{i}-\frac{v_{i}-\xi_{i}}{\left(\rho_{i} \mu_{i}+\eta_{i} \sigma_{i}\right)^{2}}\right|<\frac{\sqrt{\left(v_{i}-\xi_{i}\right)^{2}-\left(\rho_{i} \mu_{i}+\eta_{i} \sigma_{i}\right)^{2}\left(4 h_{i}\right)\left(1-h_{i}\right)}}{\left(\rho_{i} \mu_{i}+\eta_{i} \sigma_{i}\right)^{2}}, \quad i=1,2,3 ;
$$

(iii) for each $i=1,2,3$

$$
\frac{\Phi_{n, N_{i}}\left(r_{i}\right)+h_{i}}{1-h_{i}} \leq \frac{\Phi_{N_{i}}\left(r_{i}\right)+h_{i}}{1-h_{i}}<1,
$$

where

$$
\left\{\begin{array}{l}
\Phi_{N_{i}}\left(r_{i}\right)=\sqrt{1-2 r_{i}\left(v_{i}-\xi_{i}\right)+r_{i}^{2}\left(\left(\rho_{i} \mu_{i}+\eta_{i} \sigma_{i}\right)(1+M)\right)^{2}} \\
\Phi_{n, N_{i}}\left(r_{i}\right)=\sqrt{1-2 r_{i}\left(v_{i}-\xi_{i}\right)+r_{i}^{2}\left(\left(\rho_{i} \mu_{i}+\eta_{i} \sigma_{i}\right)\left(1+\epsilon_{n}\right)\right)^{2}}
\end{array}\right.
$$

where $M=\sup _{n \geq 1} \epsilon_{n}$.

(iv) $\left\{\alpha_{n}\right\} \subset(0,1)$ such that $\sum_{n=0}^{\infty} \alpha_{n}=\infty$.

Then the sequences $\left\{x_{n}\right\},\left\{y_{n}\right\},\left\{z_{n}\right\},\left\{u_{n, i}\right\},\left\{v_{n, i}\right\}$ suggested by Algorithm 1 converge strongly to $x^{*}, y^{*}, z^{*}, u_{i}^{*}, v_{i}^{*} i=1,2,3$ respectively, and $\left(x^{*}, y^{*}, z^{*}, u_{i}^{*}, v_{i}^{*}\right) \in$ $\operatorname{SNSVVID}(\Xi, \mathfrak{A}, \mathfrak{B}, \wedge, K), x^{*} \in F(S)$.

Proof. Let $\left(x^{*}, y^{*}, z^{*}, u_{i}^{*}, v_{i}^{*}\right) \in \operatorname{SNSVVID}(\Xi, \mathfrak{A}, \mathfrak{B}, \wedge, K)$ and $x^{*} \in F(S)$. By (2) and (4) we have

$$
\left\{\begin{array}{l}
g_{3}\left(z^{*}\right)=J_{\phi}^{r_{3}}\left[g_{3}\left(x^{*}\right)-r_{3} N_{3}\left(u_{3}^{*}, v_{3}^{*}\right)\right] \\
g_{2}\left(y^{*}\right)=J_{\phi}^{r_{2}}\left[g_{2}\left(z^{*}\right)-r_{2} N_{2}\left(u_{2}^{*}, v_{2}^{*}\right)\right] \\
x^{*}=\left(1-\alpha_{n}\right) x^{*}+\alpha_{n} S\left(x^{*}-g_{1}\left(x^{*}\right)+J_{\phi}^{r_{1}}\left[g_{1}\left(y^{*}\right)-r_{1} N_{1}\left(u_{1}^{*}, v_{1}^{*}\right)\right]\right)
\end{array}\right.
$$

Consequently, by (5) and (6), we have

$$
\begin{aligned}
&\left\|x_{n+1}-x^{*}\right\| \\
&=\left\|\left(1-\alpha_{n}\right) x_{n}+\alpha_{n} S\left(x_{n}-g_{1}\left(x_{n}\right)+J_{\phi}^{r_{1}}\left[g_{1}\left(y_{n}\right)-r_{1} N_{1}\left(n_{n, 1}, v_{n, 1}\right)\right]\right)-x^{*}\right\| \\
& \leq\left(1-\alpha_{n}\right)\left\|x_{n}-x^{*}\right\|+\alpha_{n} \| S\left(x_{n}-g_{1}\left(x_{n}\right)+J_{\phi}^{r_{1}}\left[g_{1}\left(y_{n}\right)-r_{1} N_{1}\left(u_{n, 1}, v_{n, 1}\right)\right]\right) \\
&-S\left(x^{*}-g_{1}\left(x^{*}\right)+J_{\phi}^{r_{1}}\left[g_{1}\left(y^{*}\right)-r_{1} N_{1}\left(u_{1}^{*}, v_{1}^{*}\right)\right]\right) \| \\
& \leq\left(1-\alpha_{n}\right)\left\|x_{n}-x^{*}\right\|+\alpha_{n} \tau\left[\left\|x_{n}-x^{*}-\left(g_{1}\left(x_{n}\right)-g_{1}\left(x^{*}\right)\right)\right\|\right. \\
&\left.+\left\|J_{\phi}^{r_{1}}\left[g_{1}\left(y_{n}\right)-r_{1} N_{1}\left(u_{n, 1}, v_{n, 1}\right)\right]-J_{\phi}^{r_{1}}\left[g_{1}\left(y^{*}\right)-r_{1} N_{1}\left(u_{1}^{*}, v_{1}^{*}\right)\right]\right\|\right] \\
& \leq\left(1-\alpha_{n}\right)\left\|x_{n}-x^{*}\right\|+\alpha_{n} \tau\left[\left\|x_{n}-x^{*}-\left(g_{1}\left(x_{n}\right)-g_{1}\left(x^{*}\right)\right)\right\|\right. \\
&\left.+\left\|y_{n}-y^{*}-\left(g_{1}\left(y_{n}\right)-g_{1}\left(y^{*}\right)\right)\right\|+\left\|y_{n}-y^{*}-r_{1}\left(N_{1}\left(u_{n, 1}, v_{n, 1}\right)-N_{1}\left(u_{1}^{*}, v_{1}^{*}\right)\right)\right\|\right] .
\end{aligned}
$$

Since $N_{1}(\cdot, \cdot)$ is $\rho_{1}$-Lipschitz continuous with respect to the first variable and $\eta_{1}$-Lipschitz continuous with respect to the second variable, and $A_{1}$ is $\mu_{1}$-Lipschitz continuous, and $B_{1}$ is $\sigma_{1}$-Lipschitz continuous, we have

$$
\begin{aligned}
& \left\|N_{1}\left(u_{n, 1}, v_{n, 1}\right)-N_{1}\left(u_{1}^{*}, v_{1}^{*}\right)\right\| \leq \rho_{1}\left\|u_{n, 1}-u_{1}^{*}\right\|+\eta_{1}\left\|v_{n, 1}-v_{1}^{*}\right\| \\
& \quad \leq \rho_{1}\left(1+\epsilon_{n}\right) \mathcal{H}\left(A_{1}\left(y_{n}\right), A_{1}\left(y^{*}\right)\right)+\eta_{1}\left(1+\epsilon_{n}\right) \mathcal{H}\left(B_{1}\left(y_{n}\right), B_{1}\left(y^{*}\right)\right) \\
& \quad \leq \rho_{1} \mu_{1}\left(1+\epsilon_{n}\right)\left\|y_{n}-y^{*}\right\|+\eta_{1} \sigma_{1}\left(1+\epsilon_{n}\right)\left\|y_{n}-y^{*}\right\| \\
& \quad \leq\left(\rho_{1} \mu_{1}+\eta_{1} \sigma_{1}\right)\left(1+\epsilon_{n}\right)\left\|y_{n}-y^{*}\right\| .
\end{aligned}
$$


Since $N_{1}$ is $A_{1}$-strongly monotone with constant $v_{1}>0$ and $B_{1}$-relaxed monotone with constant $\xi_{i}>0$, it follows from (10) that

$$
\begin{aligned}
& \left\|y_{n}-y^{*}-r_{1}\left(N_{1}\left(u_{n, 1}, v_{n, 1}\right)-N_{1}\left(u_{1}^{*}, v_{1}^{*}\right)\right)\right\|^{2} \\
= & \left\|y_{n}-y^{*}\right\|^{2}-2 r_{1}\left\langle N_{1}\left(u_{n, 1}, v_{n, 1}\right)-N_{1}\left(u_{1}^{*}, v_{1}^{*}\right), y_{n}-y^{*}\right\rangle \\
& \quad+r_{1}^{2}\left\|N_{1}\left(u_{n, 1}, v_{n, 1}\right)-N_{1}\left(u_{1}^{*}, v_{1}^{*}\right)\right\|^{2} \\
= & \left\|y_{n}-y^{*}\right\|^{2}-2 r_{1}\left\langle N_{1}\left(u_{n, 1}, v_{n, 1}\right)-N_{1}\left(u_{1}^{*}, v_{n, 1}\right), y_{n}-y^{*}\right\rangle \\
& \quad-2 r_{1}\left\langle N_{1}\left(u_{1}^{*}, v_{n, 1}\right)-N_{1}\left(u_{1}^{*}, v_{1}^{*}\right), y_{n}-y^{*}\right\rangle+r_{1}^{2}\left\|N_{1}\left(u_{n, 1}, v_{n, 1}\right)-N_{1}\left(u_{1}^{*}, v_{1}^{*}\right)\right\|^{2} \\
\leq & \left\|y_{n}-y^{*}\right\|^{2}-2 r_{1} v_{1}\left\|y_{n}-y^{*}\right\|^{2}+2 r_{1} \xi_{1}\left\|y_{n}-y^{*}\right\|^{2} \\
& \quad+r_{1}^{2}\left(\left(\rho_{1} \mu_{1}+\eta_{1} \sigma_{1}\right)\left(1+\epsilon_{n}\right)\right)^{2}\left\|y_{n}-y^{*}\right\|^{2} \\
\leq & \left(1-2 r_{1} v_{1}+2 r_{1} \xi_{1}+r_{1}^{2}\left(\left(\rho_{1} \mu_{1}+\eta_{1} \sigma_{1}\right)\left(1+\epsilon_{n}\right)\right)^{2}\right)\left\|y_{n}-y^{*}\right\|^{2}
\end{aligned}
$$

i.e.,

$$
\left\|y_{n}-y^{*}-r_{1}\left(N_{1}\left(u_{n, 1}, v_{n, 1}\right)-N_{1}\left(u_{1}^{*}, v_{1}^{*}\right)\right)\right\|^{2} \leq\left(\Phi_{n N_{1}}\left(r_{1}\right)\right)^{2}\left\|y_{n}-y^{*}\right\|^{2},
$$

where

$$
\Phi_{n, N_{1}}\left(r_{1}\right):=\sqrt{1-2 r_{1}\left(v_{1}-\xi_{1}\right)+r_{1}^{2}\left(\left(\rho_{1} \mu_{1}+\eta_{1} \sigma_{1}\right)\left(1+\epsilon_{n}\right)\right)^{2}} .
$$

Note that

$$
\begin{gathered}
\left\|y_{n}-y^{*}\right\|=\left\|y_{n}-y^{*}-\left[g_{2}\left(y_{n}\right)-g_{2}\left(y^{*}\right)\right]+\left[g_{2}\left(y_{n}\right)-g_{2}\left(y^{*}\right)\right]\right\| \\
\leq\left\|y_{n}-y^{*}-\left[g_{2}\left(y_{n}\right)-g_{2}\left(y^{*}\right)\right]\right\|+\left\|g_{2}\left(y_{n}\right)-g_{2}\left(y^{*}\right)\right\| .
\end{gathered}
$$

Since $g_{2}$ is $\lambda_{2}$-strongly monotone and $\gamma_{2}$-Lipschitz continuous mapping, we have

$$
\begin{aligned}
& \left\|y_{n}-y^{*}-\left[g_{2}\left(y_{n}\right)-g_{2}\left(y^{*}\right)\right]\right\|^{2} \\
& =\left\|y_{n}-y^{*}\right\|^{2}-2\left\langle g_{2}\left(y_{n}\right)-g_{2}\left(y^{*}\right), y_{n}-y^{*}\right\rangle+\left\|g_{2}\left(y_{n}\right)-g_{2}\left(y^{*}\right)\right\|^{2} \\
& \leq\left\|y_{n}-y^{*}\right\|^{2}-2 \lambda_{2}\left\|y_{n}-y^{*}\right\|^{2}+\gamma_{2}^{2}\left\|y_{n}-y^{*}\right\|^{2} \\
& \leq\left(1-2 \lambda_{2}+\gamma_{2}^{2}\right)\left\|y_{n}-y^{*}\right\|^{2} \\
& =\left(h_{2}\right)^{2}\left\|y_{n}-y^{*}\right\|^{2},
\end{aligned}
$$

where $h_{2}=\sqrt{1-2 \lambda_{2}+\gamma_{2}^{2}}$.

On the other hand, by (2) and (5), we have

$$
\begin{aligned}
& \left\|g_{2}\left(y_{n}\right)-g_{2}\left(y^{*}\right)\right\| \\
& =\left\|J_{\phi}^{r_{2}}\left[g_{2}\left(z_{n}\right)-r_{2} N_{2}\left(u_{n, 2}, v_{n, 2}\right)\right]-J_{\phi}^{r_{2}}\left[g_{2}\left(z^{*}\right)-r_{2} N_{2}\left(u_{2}^{*}, v_{2}^{*}\right)\right]\right\| \\
& \leq\left\|g_{2}\left(z_{n}\right)-g_{2}\left(z^{*}\right)-r_{2}\left(N_{2}\left(u_{n, 2}, v_{n, 2}\right)-N_{2}\left(u_{2}^{*}, v_{2}^{*}\right)\right)\right\| \\
& \leq\left\|z_{n}-z^{*}-\left(g_{2}\left(z_{n}\right)-g_{2}\left(z^{*}\right)\right)\right\|+\left\|z_{n}-z^{*}-r_{2}\left(N_{2}\left(u_{n, 2}, v_{n, 2}\right)-N_{2}\left(u_{2}^{*}, v_{2}^{*}\right)\right)\right\| .
\end{aligned}
$$

In view of the assumptions of $N_{2}, A_{2}, B_{2}, g_{2}$ and by using the same method as given in the proofs in (11) and (13), we can obtain that

$$
\left\|z_{n}-z^{*}-r_{2}\left(N_{2}\left(u_{n, 2}, v_{n, 2}\right)-N_{2}\left(u_{2}^{*}, v_{2}^{*}\right)\right)\right\|^{2} \leq\left(\Phi_{n, N_{2}}\left(r_{2}\right)\right)^{2}\left\|z_{n}-z^{*}\right\|^{2},
$$


where

$$
\left(\Phi_{n, N_{2}}\left(r_{2}\right)=\sqrt{1-2 r_{2}\left(v_{2}-\xi_{2}\right)+r_{2}^{2}\left(\left(\rho_{2} \mu_{2}+\eta_{2} \sigma_{2}\right)\left(1+\epsilon_{n}\right)\right)^{2}}\right.
$$

and

$$
\left\|z_{n}-z^{*}-\left(g_{2}\left(z_{n}\right)-g_{2}\left(z^{*}\right)\right)\right\|^{2} \leq\left(h_{2}\right)^{2}\left\|z_{n}-z^{*}\right\|^{2} .
$$

From (15), (16) and (14), we have

$$
\left\|g_{2}\left(y_{n}\right)-g_{2}\left(y^{*}\right)\right\| \leq\left(\Phi_{n, N_{2}}\left(r_{2}\right)+h_{2}\right)\left\|z_{n}-z^{*}\right\| .
$$

Combining (12), (13) and (17) we obtained

$$
\left\|y_{n}-y^{*}\right\| \leq h_{2}\left\|y_{n}-y^{*}\right\|+\left(\Phi_{n N_{2}}\left(r_{2}\right)+h_{2}\right)\left\|z_{n}-z^{*}\right\| .
$$

Observe that

$$
\begin{aligned}
\left\|z_{n}-z^{*}\right\| & =\left\|z_{n}-z^{*}-\left[g_{3}\left(z_{n}\right)-g_{3}\left(z^{*}\right)\right]+\left[g_{3}\left(z_{n}\right)-g_{3}\left(z^{*}\right)\right]\right\| \\
& \leq\left\|z_{n}-z^{*}-\left[g_{3}\left(z_{n}\right)-g_{3}\left(z^{*}\right)\right]\right\|+\left\|g_{3}\left(z_{n}\right)-g_{3}\left(z^{*}\right)\right\| .
\end{aligned}
$$

and in view of (2) and (5), we have

$$
\begin{aligned}
\left\|g_{3}\left(z_{n}\right)-g_{3}\left(z^{*}\right)\right\| \leq & \left\|x_{n}-x^{*}-\left[g_{3}\left(x_{n}\right)-g_{3}\left(x^{*}\right)\right]\right\| \\
& +\left\|x_{n}-x^{*}-r_{3}\left(N_{3}\left(u_{n, 3}, v_{n, 3}\right)-N_{3}\left(u_{3}^{*}, v_{3}^{*}\right)\right)\right\| .
\end{aligned}
$$

By using the assumptions on $N_{3}, A_{3}, B_{3}$ and $g_{3}$, we have

$$
\left\|x_{n}-x^{*}-r_{3}\left(N_{3}\left(u_{n, 3}, v_{n, 3}\right)-N_{3}\left(u_{3}^{*}, v_{3}^{*}\right)\right)\right\|^{2} \leq\left(\Phi_{n, N_{3}}\left(r_{3}\right)\right)^{2}\left\|x_{n}-x^{*}\right\|^{2} .
$$

where

$$
\begin{gathered}
\Phi_{n, N_{3}}\left(r_{3}\right)=\sqrt{1-2 r_{3}\left(v_{3}-\xi_{3}\right)+r_{3}^{2}\left(\left(\rho_{3} \mu_{3}+\eta_{3} \sigma_{3}\right)\left(1+\epsilon_{n}\right)\right)^{2}} \\
\left\|x_{n}-x^{*}-\left[g_{3}\left(x_{n}\right)-g_{3}\left(x^{*}\right)\right]\right\|^{2} \leq\left(h_{3}\right)^{2}\left\|x_{n}-x^{*}\right\|^{2} . \\
\left\|z_{n}-z^{*}-\left[g_{3}\left(z_{n}\right)-g_{3}\left(z^{*}\right)\right]\right\|^{2} \leq\left(h_{3}\right)^{2}\left\|z_{n}-z^{*}\right\|^{2} .
\end{gathered}
$$

Substituting (21) and (22) into (20), we have

$$
\left\|g_{3}\left(z_{n}\right)-g_{3}\left(z^{*}\right)\right\| \leq\left(\Phi_{n, N_{3}}\left(r_{3}\right)+h_{3}\right)\left\|x_{n}-x^{*}\right\| .
$$

Combining (19), (23) and (24), it yields that

$$
\left\|z_{n}-z^{*}\right\| \leq h_{3}\left\|z_{n}-z^{*}\right\|+\left(\Phi_{n, N_{3}}\left(r_{3}\right)+h_{3}\right)\left\|x_{n}-x^{*}\right\| .
$$

This imply that

$$
\left\|z_{n}-z^{*}\right\| \leq \frac{\left(\Phi_{n, N_{3}}\left(r_{3}\right)+h_{3}\right)}{1-h_{3}}\left\|x_{n}-x^{*}\right\| .
$$

Substituting (26) into (18) we have

$$
\left\|y_{n}-y^{*}\right\| \leq h_{2}\left\|y_{n}-y^{*}\right\|+\frac{\left(\Phi_{n, N_{2}}\left(r_{2}\right)+h_{2}\right)\left(\Phi_{n, N_{3}}\left(r_{3}\right)+h_{3}\right)}{1-h_{3}}\left\|x_{n}-x^{*}\right\|,
$$

that is

$$
\left\|y_{n}-y^{*}\right\| \leq \frac{\left(\Phi_{n, N_{2}}\left(r_{2}\right)+h_{2}\right)\left(\Phi_{n, N_{3}}\left(r_{3}\right)+h_{3}\right)}{\left(1-h_{2}\right)\left(1-h_{3}\right)}\left\|x_{n}-x^{*}\right\| .
$$


From (11) and (28), we get

$$
\begin{aligned}
& \left\|y_{n}-y^{*}-r_{1}\left[N_{1}\left(u_{n, 1}, v_{n, 1}\right)-N_{1}\left(u_{1}^{*}, v_{1}^{*}\right)\right]\right\| \\
& \leq \frac{\left(\Phi_{n, N_{1}}\left(r_{1}\right)\right)\left(\Phi_{n, N_{2}}\left(r_{2}\right)+h_{2}\right)\left(\Phi_{n, N_{3}}\left(r_{3}\right)+h_{3}\right)}{\left(1-h_{2}\right)\left(1-h_{3}\right)}\left\|x_{n}-x^{*}\right\| .
\end{aligned}
$$

On the other hand, since $g_{1}$ is $\lambda_{1}$-strongly monotone and $\gamma_{1}$-Lipschitz continuous mapping, we have

$$
\begin{gathered}
\left\|x_{n}-x^{*}-\left(g_{1}\left(x_{n}\right)-g_{1}\left(x^{*}\right)\right)\right\|^{2}=\left\|x_{n}-x^{*}\right\|^{2}+\left\|g_{1}\left(x_{n}\right)-g_{1}\left(x^{*}\right)\right\|^{2} \\
-2\left\langle x_{n}-x^{*}, g_{1}\left(x_{n}\right)-g_{1}\left(x^{*}\right)\right\rangle \\
\leq\left(1-2 \lambda_{1}+\gamma_{1}^{2}\right)\left\|x_{n}-x^{*}\right\|^{2}=h_{1}^{2}\left\|x_{n}-x^{*}\right\|^{2}
\end{gathered}
$$

i.e.,

$$
\left\|x_{n}-x^{*}-\left(g_{1}\left(x_{n}\right)-g_{1}\left(x^{*}\right)\right)\right\| \leq h_{1}\left\|x_{n}-x^{*}\right\| .
$$

Similarly, we have

$$
\left\|y_{n}-y^{*}-\left(g_{1}\left(y_{n}\right)-g_{1}\left(y^{*}\right)\right)\right\| \leq h_{1}\left\|y_{n}-y^{*}\right\| .
$$

Substituting (28) into (31), we have

$$
\begin{aligned}
& \left\|y_{n}-y^{*}-\left(g_{1}\left(y_{n}\right)-g_{1}\left(y^{*}\right)\right)\right\| \\
& \leq h_{1} \frac{\left(\Phi_{n, N_{2}}\left(r_{2}\right)+h_{2}\right)\left(\Phi_{n, N_{3}}\left(r_{3}\right)+h_{3}\right)}{\left(1-h_{2}\right)\left(1-h_{3}\right)}\left\|x_{n}-x^{*}\right\| .
\end{aligned}
$$

Set

$$
\ell_{n}=\frac{\left(\Phi_{n, N_{2}}\left(r_{2}\right)+h_{2}\right)\left(\Phi_{n, N_{3}}\left(r_{3}\right)+h_{3}\right)}{\left(1-h_{2}\right)\left(1-h_{3}\right)} .
$$

Substituting (30), (31), (32) and (33) into (9), we get

$$
\left\|x_{n+1}-x^{*}\right\| \leq\left(1-\alpha_{n}\left(1-\tau\left(h_{1}+h_{1} \ell_{n}+\Phi_{n, N_{1}}\left(r_{1}\right) \ell_{n}\right)\right)\right)\left\|x_{n}-x^{*}\right\| .
$$

Since

$$
\begin{aligned}
\Phi_{n, N_{i}}\left(r_{i}\right) & :=\sqrt{1-2 r_{i}\left(v_{i}-\xi_{n}\right)+r_{i}^{2}\left(\left(\rho_{i} \mu_{i}+\eta_{i} \sigma_{i}\right)\left(1+\epsilon_{n}\right)\right)^{2}} \\
& \leq \sqrt{1-2 r_{i}\left(v_{i}-\xi_{n}\right)+r_{i}^{2}\left(\left(\rho_{i} \mu_{i}+\eta_{i} \sigma_{i}\right)(1+M)\right)^{2}}:=\Phi_{N_{i}}\left(r_{i}\right),
\end{aligned}
$$

letting $\ell:=\frac{\left(\Phi_{N_{2}}\left(r_{2}\right)+h_{2}\right)\left(\Phi_{N_{3}}\left(r_{3}\right)+h_{3}\right)}{\left(1-h_{2}\right)\left(1-h_{3}\right)}$, then we have $\ell_{n} \leq \ell$. Therefore from (34) we have that

$$
\left\|x_{n+1}-x^{*}\right\| \leq\left(1-\alpha_{n}\left(1-\tau\left(h_{1}+h_{1} \ell+\Phi_{N_{1}}\left(r_{1}\right) \ell\right)\right)\right)\left\|x_{n}-x^{*}\right\| .
$$

By condition (iii)

$$
\prod_{i=1}^{3} \frac{\Phi_{N_{i}}\left(r_{i}\right)+h_{i}}{1-h_{i}}<1
$$

this imply that

$$
\ell<\frac{1-h_{1}}{\Phi_{N_{1}}\left(r_{1}\right)+h_{1}}
$$

that is

$$
\mathfrak{s}:=h_{1}+h_{1} \ell+\Phi_{N_{1}}\left(r_{1}\right) \ell<1 .
$$


Put

$$
\left\{\begin{array}{l}
a_{n}=\left\|x_{n}-x^{*}\right\| \\
t_{n}=\alpha_{n}(1-\tau \mathfrak{s}) .
\end{array}\right.
$$

By the assumption that $0<\tau \leq 1$, it follows that

$$
\tau \mathfrak{\Im} \in(0,1) .
$$

This imply that $t_{n} \in(0,1)$. From assumption (iv) we have

$$
\sum_{n=0}^{\infty} t_{n}=\infty
$$

These show that all conditions in Lemma 2 are satisfied. Hence $x_{n} \rightarrow x^{*}$ as $n \rightarrow \infty$. Consequently from (26) and (28), we have $z_{n} \rightarrow z^{*}$ and $y_{n} \rightarrow y^{*}$ as $n \rightarrow \infty$, respectively. Moreover since $A_{i}$ is $\mu_{i}$-Lipschitz continuous and $B_{i}$ is $\sigma_{i}$-Lipschitz continuous with $\mu_{i}<1, \sigma_{i}<1$, we can also prove that $\left\{u_{n, i}\right\}$ and $\left\{v_{n, i}\right\}, i=1,2,3$ are Cauchy sequences. Thus there exists $u_{i}^{*}, v_{i}^{*} \in H$ such that $u_{n, i} \rightarrow u_{i}^{*}, v_{n, i} \rightarrow v_{i}^{*}, \quad(i=1,2,3)$ as $n \rightarrow \infty$. Moreover by using the continuity of mappings $A_{i}, B_{i}, g_{i}, N_{i}, J_{\phi}^{r_{i}}, i=1,2,3$, it follows from (5) that

$$
\begin{gathered}
g_{3}\left(z^{*}\right)=J_{\phi}^{r_{3}}\left[g_{3}\left(x^{*}\right)-r_{3} N_{3}\left(u_{3}^{*}, v_{3}^{*}\right)\right], \\
g_{2}\left(y^{*}\right)=J_{\phi}^{r_{2}}\left[g_{2}\left(z^{*}\right)-r_{2} N_{2}\left(u_{2}^{*}, v_{2}^{*}\right)\right], \\
x^{*}=S\left(x^{*}-g_{1}\left(x^{*}\right)+J_{\phi}^{r_{1}}\left[g_{1}\left(y^{*}\right)-r_{1} N_{1}\left(u_{1}^{*}, v_{1}^{*}\right)\right]\right) .
\end{gathered}
$$

Hence from Lemma 2 it follows that $\left(x^{*}, y^{*}, z^{*}, u_{i}^{*}, v_{i}^{*}\right) \in \operatorname{SNSVVID}(\Xi, \mathfrak{A}, \mathfrak{B}, \wedge, K)$. Finally we prove that $u_{i}^{*} \in A_{i}\left(y^{*}\right)$ and $v_{i}^{*} \in B_{1}\left(y^{*}\right)$ Indeed we have

$$
\begin{aligned}
d\left(u_{1}^{*}, A_{1}\left(y^{*}\right)\right) & =\inf \left\{\left\|u_{1}^{*}-w\right\|: w \in A_{1}\left(y^{*}\right)\right\} \\
& \leq\left\|u_{1}^{*}-u_{n, 1}\right\|+d\left(u_{n, 1}, A_{1}\left(y^{*}\right)\right) \\
& \leq\left\|u_{1}^{*}-u_{n, 1}\right\|+\mathcal{H}\left(A_{1}\left(y_{n}\right), A_{1}\left(y^{*}\right)\right) \\
& \leq\left\|u_{1}^{*}-u_{n, 1}\right\|+\mu_{1}\left\|y_{n}-y^{*}\right\| \rightarrow 0 \text { as } n \rightarrow \infty .
\end{aligned}
$$

That is $d\left(u_{1}^{*}, A_{1}\left(y^{*}\right)\right)=0$. Since $A_{1}\left(y^{*}\right) \in C B(H)$, we must have $u_{1}^{*} \in A_{1}\left(y^{*}\right)$. Similarly we can show that $u_{2}^{*} \in A_{2}\left(z^{*}\right), u_{3}^{*} \in A_{3}\left(x^{*}\right), v_{1}^{*} \in B_{1}\left(y^{*}\right), v_{2}^{*} \in B_{2}\left(z^{*}\right)$ and $v_{3}^{*} \in B_{3}\left(x^{*}\right)$. This complete the proof.

\section{Competing interests}

The authors declare that they have no competing interests.

\section{Authors' contributions}

All authors read and approved the final manuscript.

\section{Acknowledgements}

This work was supported by the National Natural Science Foundation of China (Grant No.11361070).

\section{Author details}

${ }^{1}$ College of Statistics and Mathematics, Yunnan University of Finance and Economics, Kunming, Yunnan 650221, China.

${ }^{2}$ Department of Mathematics, Jazan University, Jazan, Kingdom of Saudi Arabia. 
Received: 10 April 2014 Accepted: 5 June 2014

Published: 25 June 2014

\section{References}

Agarwal RP, Cho YJ, Petrot N (2011) System of general nonlinear set valued mixed variational inequality problems in Hilbert spaces. Fixed Point Theory Appl 2011(31):10

Ahmad MK, Salahuddin S (2012) Stable perturbed algorithms for a new class of generalized nonlinear implicit quasi variational inclusions in Banach spaces. Adv Pure Math 2(3):139-148

Aubin JP (1982) Mathematical Methods of game theory and economics. North Holland, Amsterdam, The Netherlands

Baiocchi C, Capelo A (1984) Variational and Quasi variational inequalities. Applications to Free Boundary Problems, John Wiley and Sons, New York

Brezis H (1973) Operateurs maximaux monotone er semi groupes de contractions dans les espaces de Hilbert. North-Holland Mathematices Studies 5 Notes de Matematica (50) North-Holland, Amsterdam

Chang SS (1984) Fixed point theory with applications. Chongqing Publishing House, Chongqing

Chang SS, Lee HWJ, Chan CK (2007) Generalized system for relaxed cocoercive variational inequalities in Hilbert spaces. Appl Math Lett 20(3):329-334

Ding XP, Luo CL (2000) Perturbed proximal point algorithms for generalized quasi variational like inclusions. J Comput Appl Math 113:153-165

Fang YP, Huang NJ, Thompson HB (2005) A new system of variational inclusisons with $(H, \eta)$-monotone operators in Hilbert spaces. Comput Math Appl 49:365-374

Giannessi F, Maugeri A (1995) Variational inequalities and network equilibrium problems. Plenum Press, New York NY USA

Hassouni A, Moudafi A (2001) A perturbed algorithms for variational inequalities. J Math Anal Appl 185:706-712

Inchan I, Petrot N (2011) System of general variational inequalities involving different nonlinear operators related to fixed point problems and its applications. Fixed Point Theory. 2011: 17, Article ID 689478, doi:10.1155/2011/689478

Kassay G, Kolumban J (2000) System of multivalued variational inequalities. Publ Mathematicae Debrecen 56(1-2): 185-195

Kim JK, Kim DS (2004) A new system of generalized nonlinear mixed variational inequalities in Hilbert spaces. J Convex Anal 11(1):235-243

Kim TH, Xu HK (2008) Convergence of the modified Mann's iterative method for asymptotically strict pseudo-contractive mappings. Nonlinear Anal Theory Methods Appl 68(9):2828-2836

Nadler SBJr (1969) Multivalued contraction mappings. Pacific J Math 30:475-487

Nie H, Liu Z, Kim KH, Kang SM (2003) A system of nonlinear variational inequalities strongly monotone and pseudo contractive mappings. Adv Nonlinear Var Inequal 6(2):91-99

Petrot N (2010) A resolvent operator technique for approximate solving of generalized system mixed variational inequalities and fixed point problems. Appl Math Lett 23(4):440-445

Suantai S, Petrot N (2011) Existence and stability of iterative algorithms for the system of nonlinear quasi mixed equilibrium problems. Appl Math Lett 24:308-313

Verma RU (1999) On a new system of nonlinear variational inequalities and associated iterative algorithms. Math-Sci Res Hotline 3(8):65-68

Verma, RU (2001a) Iterative algorithms and a new system of nonlinear quasivariational inequalities. Adv Nonlinear Var Inequal 4(1):117-124

Verma RU (2001 b) Projection methods, algorithm and a new system of nonlinear variational inequalities. Comput Math Appl 41(7-8): 1023-1031

Verma, RU (2002) Projection methods and a new system of cocoercive variational inequality problems. Inter. J Diff Equ Appl 6(4):359-367

Weng X (1991) Fixed point iteration for local strictly pseudo contractive mapping. Proc Am Math Soc 113(3):727-737

doi:10.1186/2193-1801-3-318

Cite this article as: Tang et al:: A system of nonlinear set valued variational inclusions. SpringerPlus 2014 3:318.

\section{Submit your manuscript to a SpringerOpen ${ }^{\circ}$ journal and benefit from:}

- Convenient online submission

Rigorous peer review

- Immediate publication on acceptance

- Open access: articles freely available online

- High visibility within the field

- Retaining the copyright to your article

Submit your next manuscript at $\boldsymbol{\triangleright}$ springeropen.com 